\title{
Intranet-Based Wiki with Instant Messaging Protocol
}

\author{
Chimezie F. Ugwu, Henry O. Osuagwu, and Donatus I. Bayem
}

\begin{abstract}
This research developed an Intranet-Based Wiki with Instant Messaging Protocol (IBWIMP) for the Staff of the Department of Computer Science, University of Nigeria, Nsukka to enable them to collaborate on tasks; like writing of documents like, memo, project guidelines, proposal/grants, and circulars with online security consideration. The essence of this work is to improve on the contributions staff make during work, in carrying out tasks with their colleagues irrespective of the person's location at that point in time. The existing system on ground would always require the presence of the staff within the department before they can carry out the tasks meant for them or respond to the available mails within their mail boxes located within the general office of the department. In regard to such situation, there is always delay in the processing of such mails or documents that could require urgent attention of the supposed staff therefore could cause serious damages. This research established a better internet connection for the security of the system and the documents therein by the use of virtual private server (VPS) hosting on virtual private network (VPN). This system allows the collaboration between the staff of the department and external persons, or partners classified as external staff user on documents like circulars that normally come from outside the department. This system automatically sends emails to the supposed users whenever the admin posts a document via the Short Message Transfer Protocol (SMTP). The system is accessed online by the users from any location once there is accessible internet connection and users can collaborate on the development of any posted document at the same time. This application was designed using Object Oriented Analysis and Design Methodology (OOADM) and implemented using Hypertext Markup Language (HTML), JavaScript, Cascading Style Sheet (CSS), CkEditor, Hypertext PreProcessor (PHP) and MySQL database management system.
\end{abstract}

Index Terms - Intranet, Message, VPN, VPS, Wiki.

\section{INTRODUCTION}

With the growing activities in both the academic and business world comes a growing amount of information: manuals, procedures, best practices, ideas, important documents, sales leads, expenses etc. Organizing all this information and processing all these documents increasingly becomes more work. It might be a small academic department or a small business organization, but simply using notes and paper documents is less convenient for the staff, and spending all this time on sharing information by the use of manual mail boxes known as "pigeon holes" and finding back information in file repository in the file cabinets is a huge waste of time and energy.

The absence of administrative staff in the office puts a hold on the workflow, in the sense that some documents to be

Submitted on June 24, 2021

Published on July 16, 2021.

Chimezie F. Ugwu, Institute of Management and Technology, Enugu, Nigeria.

(e-mail: chimeziefredricku@ ${ }^{\circledR}$ gmail.com) moved from a location in the office to another, in the name of information dissemination, remain unmoved. As a result of these, there could be damages depending on the need for which the information is meant to meet at that moment. The motivation for organizations to use social media has been increasing in recent times. This is because the growth of social media has become a major technological evolution that transforms the way individuals and groups work and interact together [1]. Therefore, the introduction of the use of a Wiki that is Intranet-Based with an Instant Messaging Protocol will enhance the throughput of the performance of the staff in such an environment, thereby increasing their throughput on the delivery of quality services to their clients.

A wiki will enable the staff to easily disseminate information. This application offers information sharing and collaboration features, acting as cognitive reflection and intensification tools and aiding the construction of meaning through the self-design of knowledge repositories [2]. The benefit of such technologies for corporate intranets therefore lies in their ability to build on the knowledge and information within an organization. This application will enable the staff to easily disseminate information. The application will equally grant the staff of the organization the enablement of collaborating with themselves during work from any given location. This means that, when a staff is not present in the organization, he or she stands the chances of contributing to the work going on within the organization from wherever he or she may be. This application will be designed to run on an intranet network. An intranet is a computer network that uses internet protocol technology to share information, operational systems, or computing services within an organization.

\section{REVIEW OF RELATED LITERATURE}

A wiki is a website with database developed for collaborative use by a community of users, allowing any user to add and edit content. The term is based on the Hawaiian word "wikiwiki," which means "fast, speedy; to hurry, hasten; quick, fast, swift," as defined by Bo Leuf and Ward Cunningham in their work titled "The Wiki Way: Quick Collaboration" on the web [3], whereas intranet is the generic term for a collection of private computer networks within an organization. An intranet uses network technologies as a tool to facilitate communication between people or work groups to improve the data sharing capability and overall knowledge base of an organization's employees [4].

According to the author in [3], the era of the web 2.0 was what created the enabling environment for the development

Henry O. Osuagwu, Nigeria Maritime University, Delta State, Nigeria. (corresponding e-mail: henry.osuagwu.pg68120@unn.edu.ng)

Donatus I. Bayem, Nigeria Maritime University, Delta State, Nigeria (e-mail: dbayem@ gmail.com) 
of the dynamic and collaboration-conducive websites or web applications. By Web 2.0 we mean the second generation of the World Wide Web that focused on the ability for people to collaborate online (see the advantages and disadvantages of wiki to any organization in [5]). The author in [6] attempts to investigate how Web 2.0 technologies influence workplace learning with keen interest on using Wiki as a tool for corporate exchange of knowledge. In [7], it was reviewed that Wiki pages demonstrate a conversational knowledge management solution. Conversational knowledge management has surfaced as a method for organizational knowledge creation, particularly in a virtual team environment. The prospect of several key benefits makes these solutions a favorable business option since they are not economically or technologically demanding, are quick to create knowledge and are well suited for decentralized environments.

According to the authors in [8]-[9], "Social software (wiki) has proven valuable in enterprises for collaborative knowledge management." In order to introduce a wiki in the enterprise, a solution that combines Web 2.0 and Semantic Web technologies was proposed. The authors described how the solution could be implored using a concrete enterprise scenario on proposal writing. The authors in [10]-[12], strongly believe that the process of knowledge sharing can be greatly facilitated by the use of information technology. They pointed that, successful knowledge management depends on the contributions from all the organizational members, and for the intranet-based wiki to serve as a knowledge sharing environment, high participation is important.

Gbola Olasina [13], assert that many of the knowledge management projects that commenced in the past were primarily driven by the adoption of technologies. Technologies include tools such as search engines, retrieval and classification tools, e-collaboration tools, portals and content management systems. One of the lessons learnt from these failures is that technology alone should not be the primary driver for any Knowledge Management projects/initiatives and that an appropriate balance of technology, process, people and content is instrumental to the continued success of any knowledge management deployment. In [10], the author asserts that "a fundamental knowledge sharing is the ability to share and/ or transfer knowledge within an organization and amongst its members." It was made clear that there are indeed many different collaboration and communication technologies that let users contribute information over the web, but they all enforce structure of some sort. The IBWIMP is being developed as a server-side technology with a functional database management system that will be based on the ideas of its users and presupposes the users themselves to agree upon and maintain a working structure. Some other existing wiki frameworks like "dokuwiki," has no server side technology used during its development, it architecture is just of the presentation tier. This is why the "dokuwiki" is open to anybody and prone to the risk of having its contents edited with wrong information.

According to [14], "the rapid development of the intrawebs was as a result of the realization of the internal use of the web technology by some business organizations." The author in that paper presented what is known as the wiki concept, focusing its uses on the intranet, in both the academic and business organizations. From that paper, it is learnt that at about the year 2000, intranet-based wiki was developed and installed in the Computer Science Department of the University of Nice, France. It is used by all the teachers, students, industrial partners, etc. Before then, at about 1999, ILOG Company had developed a very impressive wikicentered intranet. At about the same period of time, other companies like Google, Motorola, and New York Times Digital started making publicly the ways they use wiki in their organizations which depicted the general acceptance of the technology [13].

In the year 2013, the study in [15] was carried out in the Department of Computer Science, University of Nigeria, Nsukka. The author presented the work as an online system that could be used by the staff to receive information on decision making processes within the department. This is basically achieved by the staff responding to memo posted on the system by the head of the department. Other characteristics featured in the system are the system could be used to assign project topics to students, it presented the staff mail box online to enable them access their mails from different locations. In its downsides or limitations according to the author, users (staff) are to go online and check for posts or mails posted on the system from time to time, the head of the department happens to be the one acting as the admin and there was little or no emphasis on the security of the information contained in the system. But in the proposed system (IBWIMP), it is presented as online system basically meant for knowledge sharing, that is to be hosted in a virtual private server (VPS) which must be on a virtual private network (VPN), in order to prevent the intrusion of hackers, which makes it an intranet-based system. The system has four categories of users with different levels of privileges; the admin category- and must not be the head of the department, the staff category, the external staff category- partners from other departments or offices outside, and the student category- student within the department. The proposed system allows the staff to collaborate on the writing of many important documents like memo, circulars, proposal/grant, and project guideline, thereby constituting the four classes of information documents that can be collaboratively written on it.

The proposed system improves the cited work above by its ability to send email alert or notifications to the supposed users whenever information or a document is posted on the system via a short message transfer protocol (SMTP), irrespective of the users' location and this makes the system less personnel intensive and less time consuming. The proposed system enables users to collaborate on the manipulation of images and texts, and this makes the system similar to the "visual wiki" in [16] by the use of CkEditor that allows the attachment of pictures on the web pages or web documents. And it provides the users with comment text fields to prevent the loss of the originality or authenticity contents by continuous modification of contents by users.

The study in [17] found several benefits of the use of intranet-based wiki. The author concluded that it improved the work conditions in some projects and even made some projects possible. It enabled people to participate in internal communication and collaborative projects, share information, 
ask other people to share information, and manage work in a meaningful way. It had become a vital part of the daily work for many. According to the authors in [5], "Wikis have been revolutionary in a wide variety of institutional contexts, including educational institutions, research centers, security agencies, and business corporations". It was mentioned in [5] that, "Enterprises that rely heavily on employees with high intelligence and hands-on experience - NASA (National Aeronautics and Space Administration), Northrop Grumman (Security Agency), the CIA (Central Intelligence Agency), etc. - have also benefited from the increasing use of wikis." The case of the National Broadcasting Company (NBC in America) was illustrated in [5], which showed how the company's SED (Software Engineering and Delivery) team moved from the use of a system known as support central that was designed for workflow and collaboration management, which enhanced business processes and knowledge sharing to what was initially known as confluence wiki that provided the team with the communication platform they needed among its programmers in dispersed location. Other company operating alongside the NBC like the Peacock production (a media firm in America) also went into developing wiki for use in carrying out their daily operations especially among a specified group of persons.

In this review, the experience acquired is that, the reasons behind the development of an intranet-based wiki, in most of the already published works, are almost the same. The authors sited in this review presented the driving force for the development of an intranet-based wiki as it being "a tool for collaboration" during work or while carrying out tasks or assignments. The limitations of other systems existing before the development of the intranet-based wiki (like the Support Central System used NBC in [5] and the wiki system in [15]) were the inability of those systems to reach out to their users who could be in dispersed geographic locations when there is need for a collaborative task to be carried out and its inability to send notifications to users whenever information is posted by the admin. These are the reasons why the development of IBWIMP became necessary in this research to help build a collaborative teamwork spirit, and in addition, to facilitate knowledge sharing among the Academic Staff of the Department of Computer Science, University of Nigeria, Nsukka (UNN), which do not disagree with the aim and objectives of this work. The intranet-based nature of the proposed system makes it completely different from other wikis already in use, like the dokuwiki framework in the sense that according to the scope of this work, its users are mainly the staff of the computer science department, UNN, while the other wiki frameworks have no limit to the number of users. The dokuwiki and some other wiki frameworks were developed without database, while in the development of this work, database is considered very indispensable. Authorization of users via user registration by the system administrator enables the system to check users' access, and this feature is not found in most of the wiki frameworks in use today. The successful planning of this work is based on the Nexus model of an intranet application in [18].

\section{SySTEM ANALYSIS DESIGN}

This section presents a detailed analysis of the IBWIMP and Object Oriented Analysis and Design Methodology (OOADM) as the design methodology used in the development of this work. The OOADM is preferable to other system analysis and design methodologies that can be used in the field of software engineering. The object oriented analysis and design is a technical approach for analyzing and designing an application or system by applying the object oriented paradigm and visual modeling throughout the development of a system. The object oriented paradigm is based on the concept of "objects" (data structures) which contain data in the form of fields, often known as attributes. The methods are the operations that can be performed by the objects, and these operations are attributed to a given class to which a set of objects belong. The visual modeling used in this work is unified modeling language (UML), which is a standard language for visualizing, specifying constructing and documenting the components of a software system during it development [17]. Among the various modeling styles that UML consists of, the following will be used in the design of the proposed system:

- The use case diagram;

- The class diagram.

\section{A. Description of the Existing System}

The existing system of information sharing among the staff in the department of computer science, UNN, is a manualbased system. In this system, documents or files (for example, memo or circular) are manually transferred from one staff to another by a clerk on duty. When a staff is not on seat, such documents or file meant for the staff is drop in his or her manual mail box (pigeon holes), usually placed in the general office within the department. Most times, such documents could need an urgent attention, but when the staff is not on seat, the process gets delayed pending when the staff returns. This delay being encountered among the members of the staff leads to a slow mode of operation in carrying out some very important tasks that could be dependent of time.

Not only the delay, the confidentiality of the information on such documents or files could be compromised, in the sense that the manual mail boxes in which the documents are dropped are always open and it could be accessed by anybody within the reach. At such, the documents could be mutilated, stolen, duplicated without the consent of its owner and therefore could lack integrity or even have its content delete or destroyed depending on individual interests among fellow staff. "Time" they say "is money." When an organization lags on meeting up with deadlines in a decision making process or execution of tasks by its employees as a result of communication gaps or inadequate means of communication, it could lead to a serious backdrop in its level of productivity.

After carefully analyzing the existing system, the following problems were identified:

- Manual system of information sharing is very stressful.

- It is risky because when such important document is dropped in the manual mail box, it could be tampered with.

- The system gives no room for collaboration among staff in carrying out task. 
- The whole process is time consuming due to the delay being encountered when a staff whose attention could be needed on an ongoing process is not on seat.

- Important information could be lost at the long run due to lack of a database management system from which it could be retrieved if need be.

\section{B. Analysis of the Proposed System}

The proposed system is to be designed to solve all the problems identified in the existing system by adhering to use of the Nexus model of an intranet in [16]. The following are the features of the proposed system:

- The proposed system has an administrator whose job is to extensively manage, maintain and regulate the functioning of the system. The admin could be a nonacademic staff but from within the department.

- It allows for only but three categories of users that must be duly registered by the admin. The categories are the staff user (academic staff within the department), the external staff user (academic or none academic staff from outside the department) and the student (selected few among the students in the department, for example, P.G Class Reps or Final year Class Rep, or the President of the departmental student association like NACOSS). After registration, users can log in with their email and password. And a user must have a profile.

- The contents to be posted on the system are of four categories: the memo (from within the department), student project guideline (from within), proposal/grant (from within) and circular (from outside the department).

- All post is to be made by the administrator as well as deletion of contents.

- The memo contents can be viewed and edited by any user of the staff user category.

- The project guideline contents can only be viewed by user of the student category, but such contents can be viewed and edited by any user of the staff user category.

- The proposal/grant contents can be viewed and edited by any user of the staff user category.

- The circular contents can be viewed and edited by any user of staff user category and external staff user category.

- When a post is made, the system automatically sends email notifications to users in the user category that is supposed to access such content category. This is achieved via SMTP (short message transfer protocol).

- Users can equally make contributions or suggestions on any post that they can view via the comment text field.

Having analyzed the features of the proposed system, it is quite obvious that the system will provide an enabling platform for its target users to collaborate.

\section{System Requirement Specification}

The design tool used in this work is UML which help the developer to properly visualize, specify, construct and document all the components that make up the system, during the design stage. The UML tools used are the use case diagram and the class diagram. Below are the diagrams:

- Class Diagram of the Intranet-Based Wiki: the class diagram of the intranet-based wiki shown in Fig. 1 present an overview of the proposed system by describing the object classes, their attributes, operations (methods) that can be perform on them, and the relationships between them within the system.

- Use Case Diagram of the Intranet-Based Wiki: this presents a picture of the interactions among the actors (users) of the proposed system and the actions (operations) that those actors can perform on the system, shown in Fig. 2.

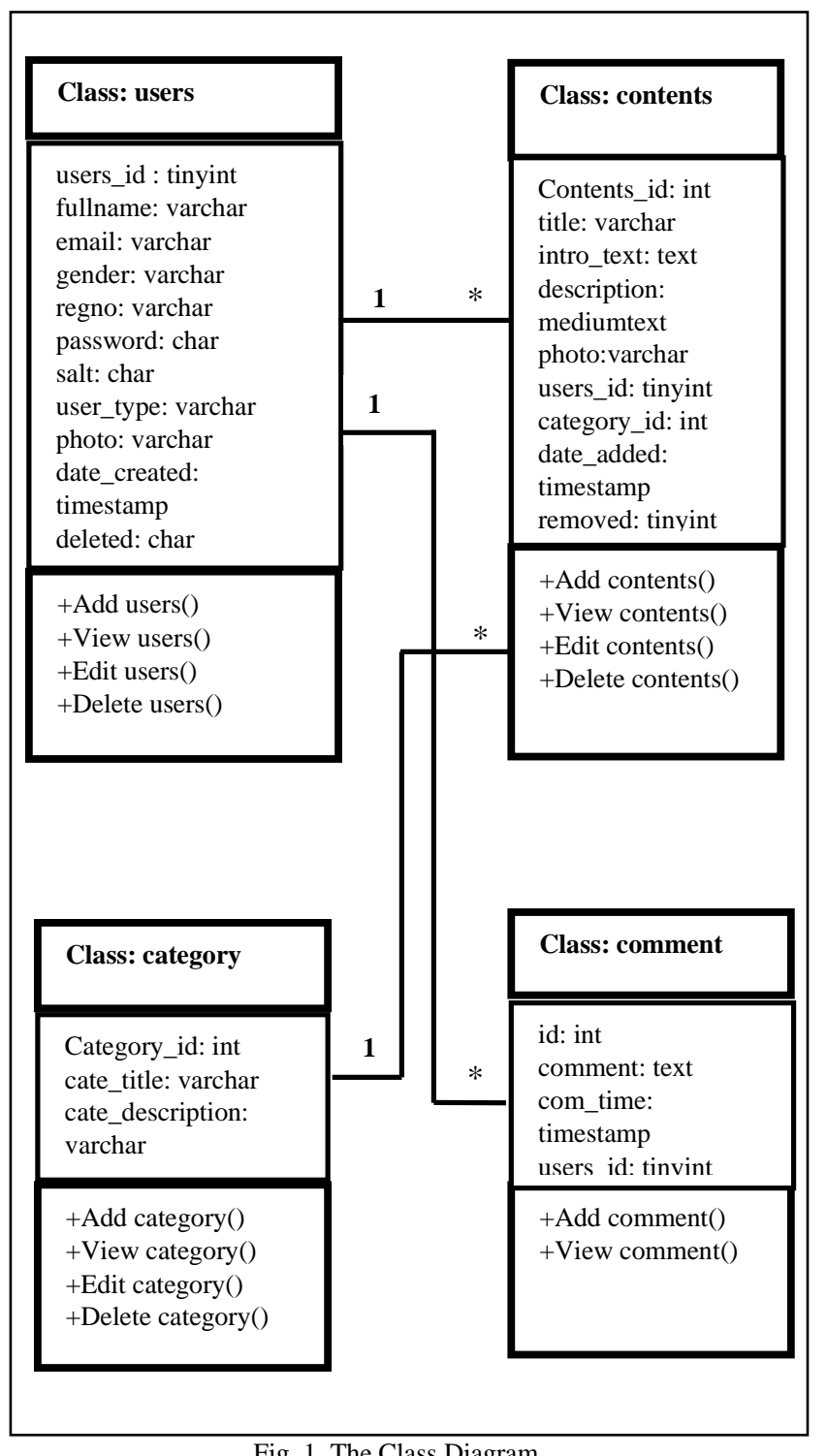

\section{Design of the Proposed System}

This section presents the necessary designs used in the development of the proposed system.

\section{Database Design}

The database management system used in this work is MYSQL. And the name of the database created for this work is "wikidb," which contains the following tables: users table, contents table, categories table and comment table.

\section{Input Design}

This refers to the design of the medium through which all the data that the system needs can be supplied to the system, with an explicit declaration of their data types. This design is basically "form design" which contains the users' data that could be needed for the system to carryout specified 
operations or tasks. Fig. 3 is the illustration of the input design of the user registration form.

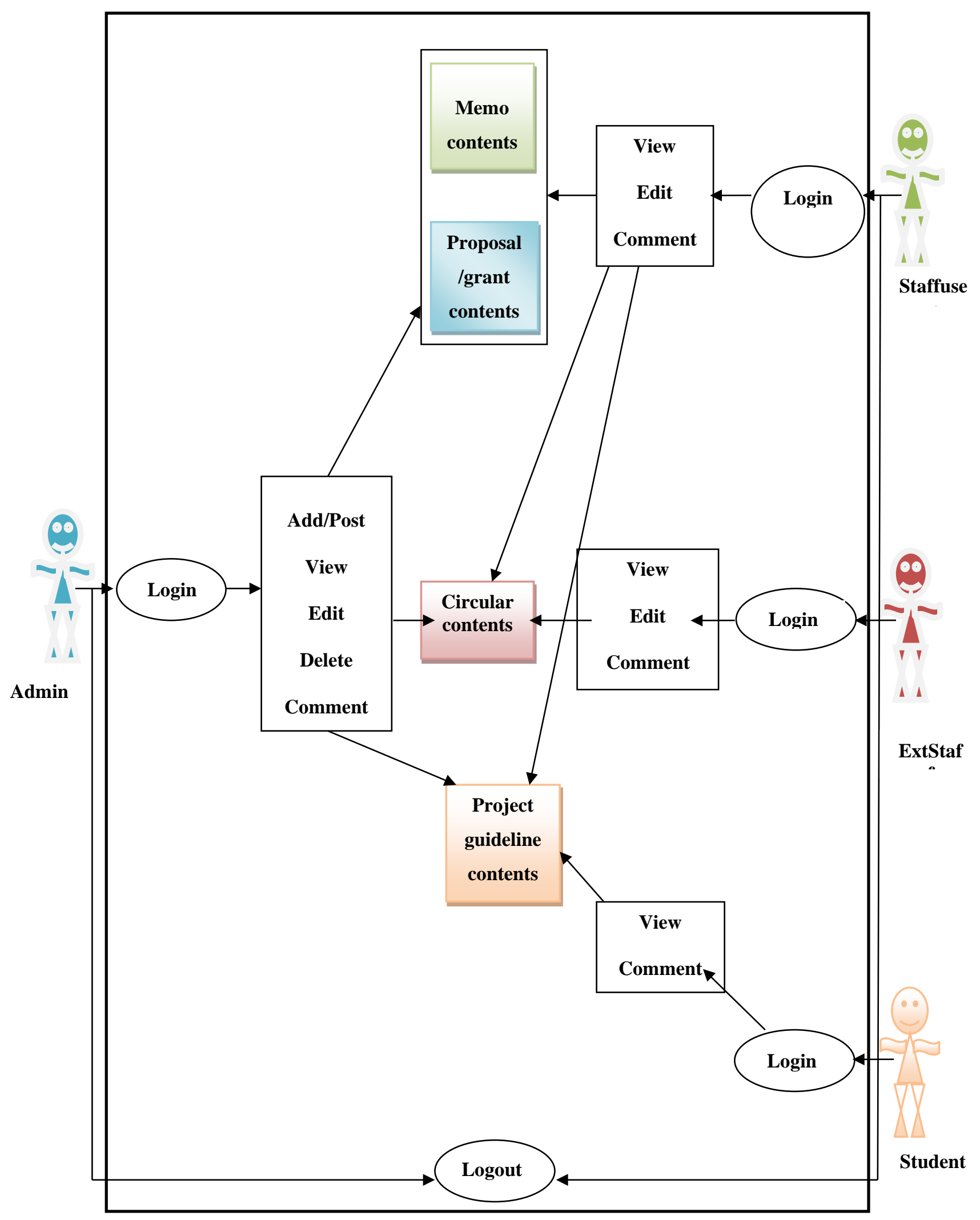

Fig. 2 The use Case Diagram 


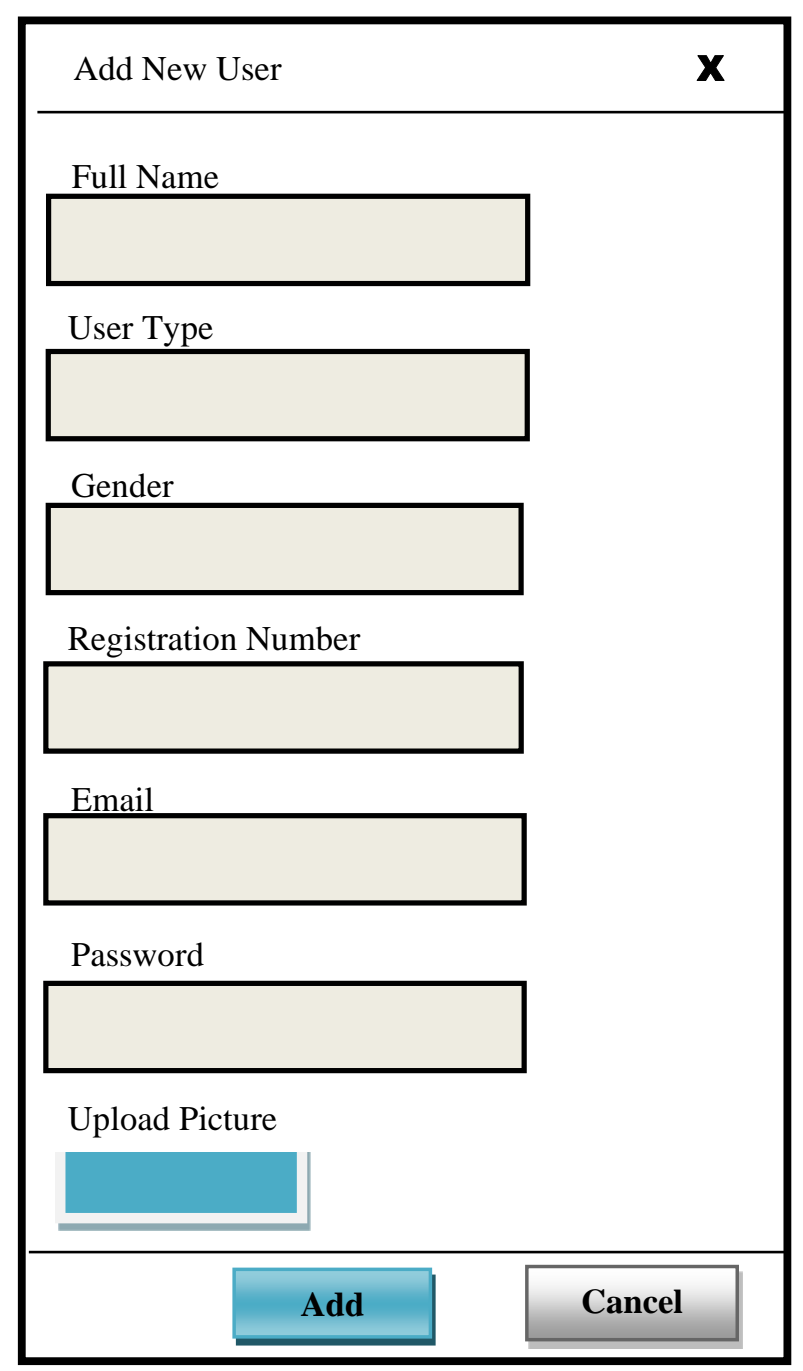

Fig. 3. Illustration of User Registration Input Design.

\section{Output Design}

The output design determines how the processed data is to be displayed. The design presented in Fig. 4 shows exactly how the user information will be display in the user profile page after each user registration.

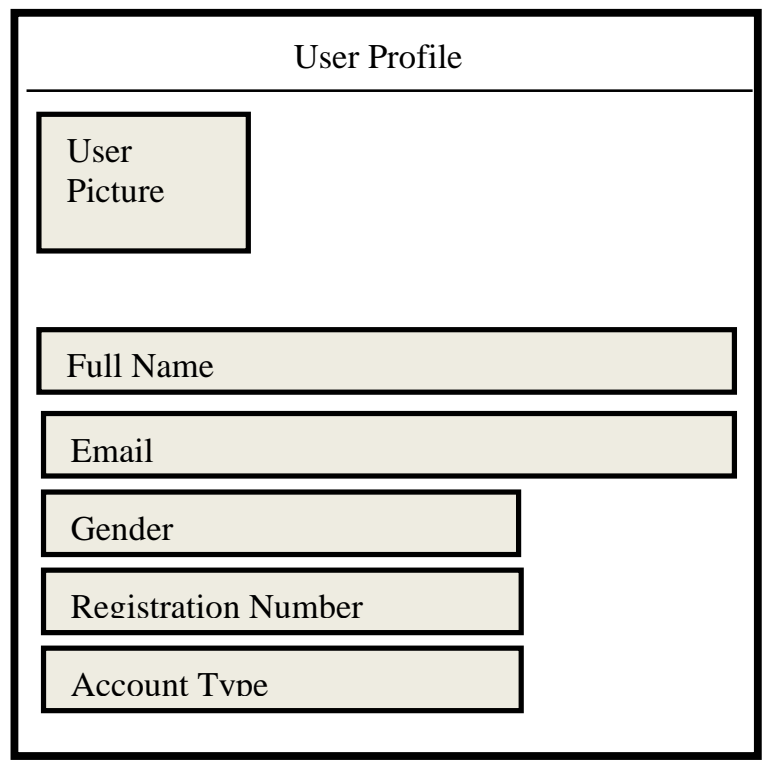

Fig. 4. Illustration of the Output Design of the user profile.

\section{The Algorithm/Flowchart of the System}

This is the graphical representation of the flow of activities within the intranet-based wiki. It shows the processes involved in operating the system by the different categories of users that are allowed to work with the system.

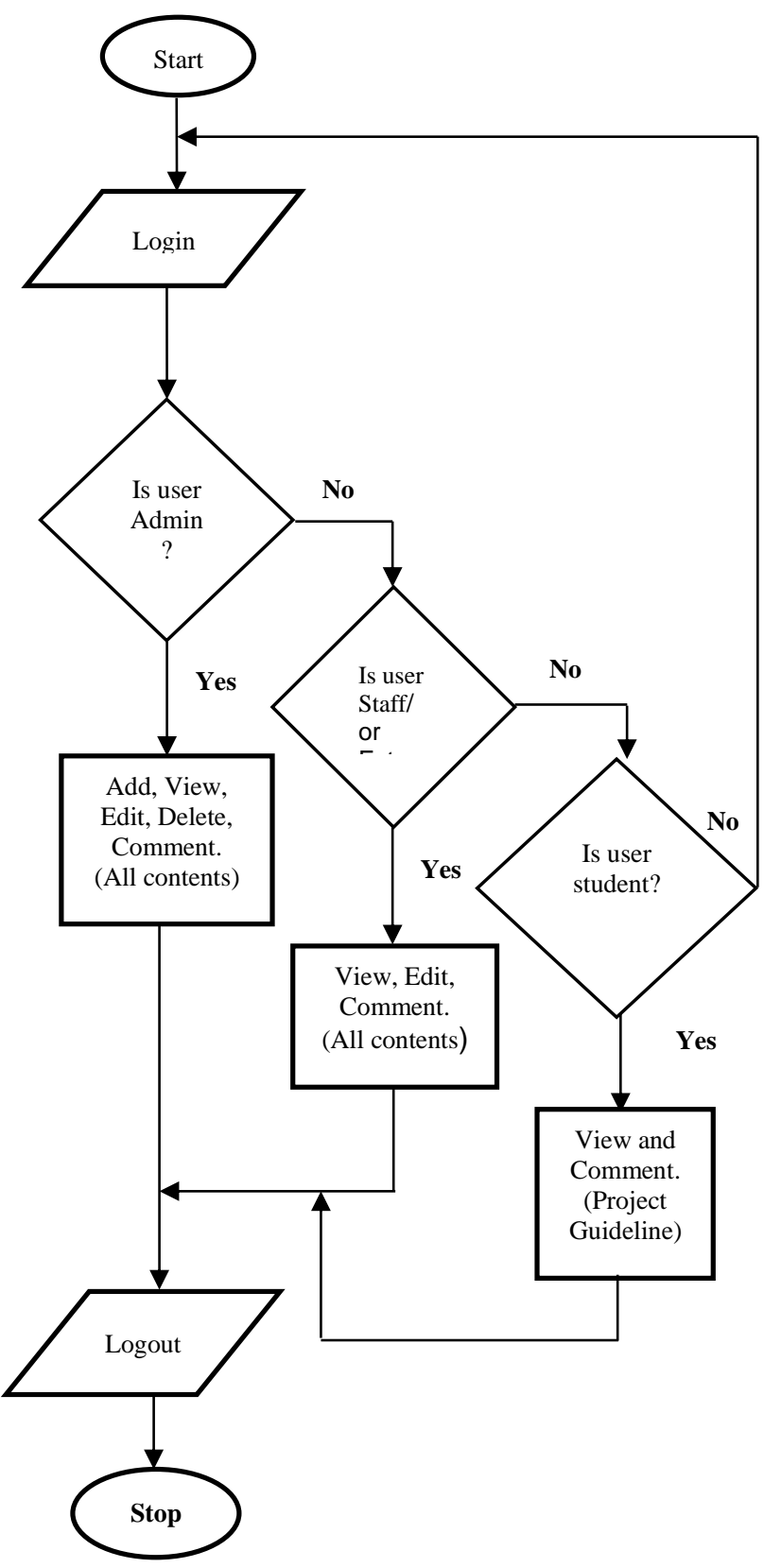

Fig. 5. The Algorithm/Flowchart design of the system.

\section{System Architecture}

The architectural design of the intranet-based wiki is of 3 (three) tiers as shown in Fig. 6. The tiers are the presentation, middle and data. The presentation tier is made up of the HTML, CSS, and JavaScript. They are used for the front end designs which include document structuring and styling. The middle tier comprises of the PHP code, which connects the presentation tier and the data tier. It is also in this middle tier that the firewall of the intranet, on which this wiki is based, is established for proper security of user data. The third, the data tier is for the database design, which serves as data repository which is needed by the system for processing of information. The database management system used for the 
design of this tier is MySQL. The middle and data tiers reside and function within the system server.

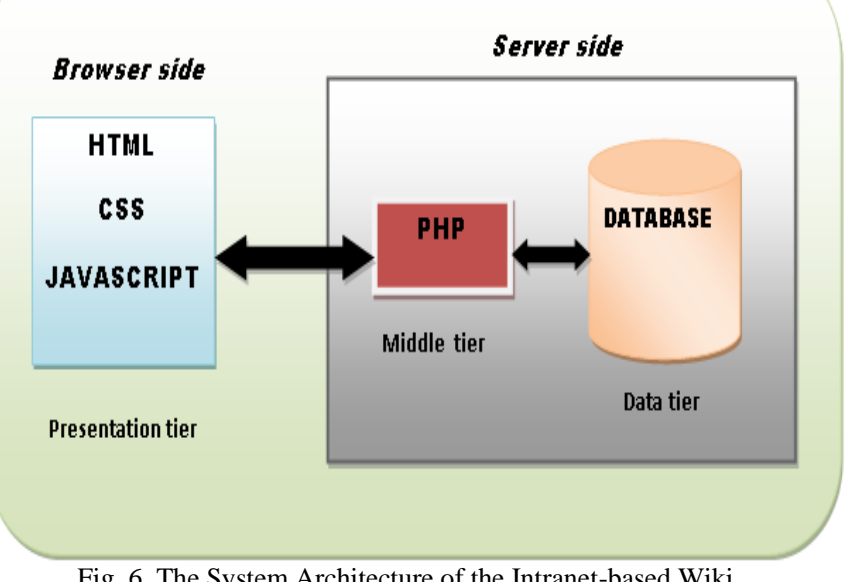

\section{Network Architecture}

In this research, the network connection for the implementation of the intranet on which the wiki is based is a VPN (Virtual Private Network) connection. The VPN is a type of network used to connect components to network by using another network like the internet. This is done by tunneling through the internet or another public network in a manner that provides the same security and features as a private network. In Fig. 7, the users are given authorization by the admin, by configuring the I.P (internet protocol) addresses of the users' systems or devices to enable access to the VPN server. The VPN server secures the wiki from being accessed by any user who is not authorized by tunneling all the configured users' connection to it secured route. This completely prevents hackers' attack on the system. Below is the Network architecture for connecting to the server in which the Wiki resides.

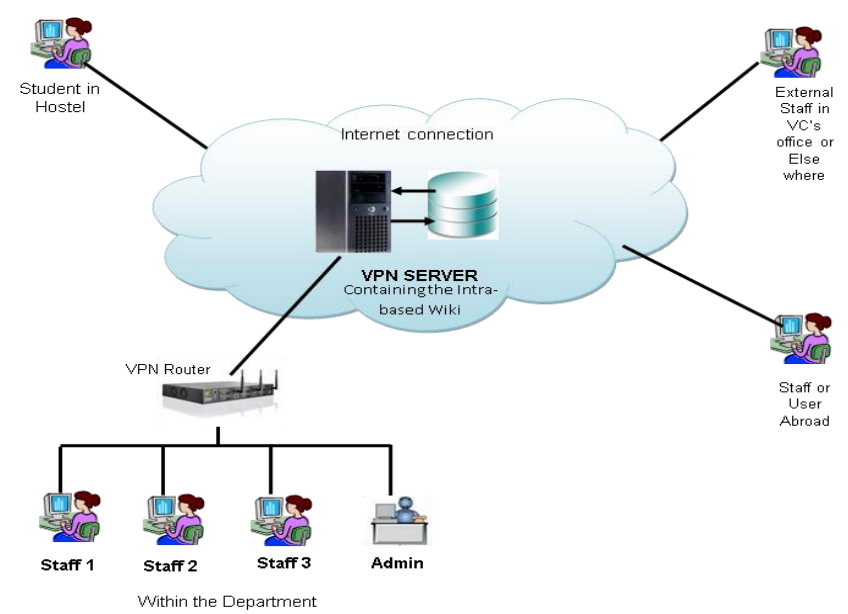

Fig. 7. The Network Architecture of the Intranet-based Wiki.

\section{SYSTEM IMPLEMENTATION}

The integrated development environment (IDE) used in the development of this research is the komodo editor 6.0, on which the source codes are written and tested on the xamp server which is used as local host server on the Microsoft windows 7 platform. Chrome, Firefox, or Torch are the browser which were used for running the intranet-based wiki. The programming languages employed in this research are HTML, CSS, JavaScript, and PHP, while MySQL database management system was used. And CKEditor is used for the input and editing of data in the system.

\section{A. Software Testing}

This system has undergone series of test during the development stage in order to detect compilation errors and have them removed. The testing is of two phases:

- Firstly, testing done during the development phases in order to remove runtime errors or bugs.

- Secondly, testing done by running the wiki on the local host sever of the computer. This is done by browsing the wiki using any of the available browsers to know if it meets the requirements of the targeted users before hosting it online, on the intranet.

In addition, every user is enjoined to stick to the steps below for smooth running of this application:

1. Ensure that the device in use is duly or properly configured by the admin with the VPN (remember that this is what makes it intranet-based for security purpose).

2. Connect to the VPN connection in your network connection panel on the right of the task bar.

After the connection, open a functional web browser on your system and type the domain name of the system on the URL (uniform resource locator) of the browser and press the enter key on your keyboard. We then have the home page as shown in Fig. 8, on which you will find the authorized user sign in button by the top right of the screen, when clicked, takes you to the login page.

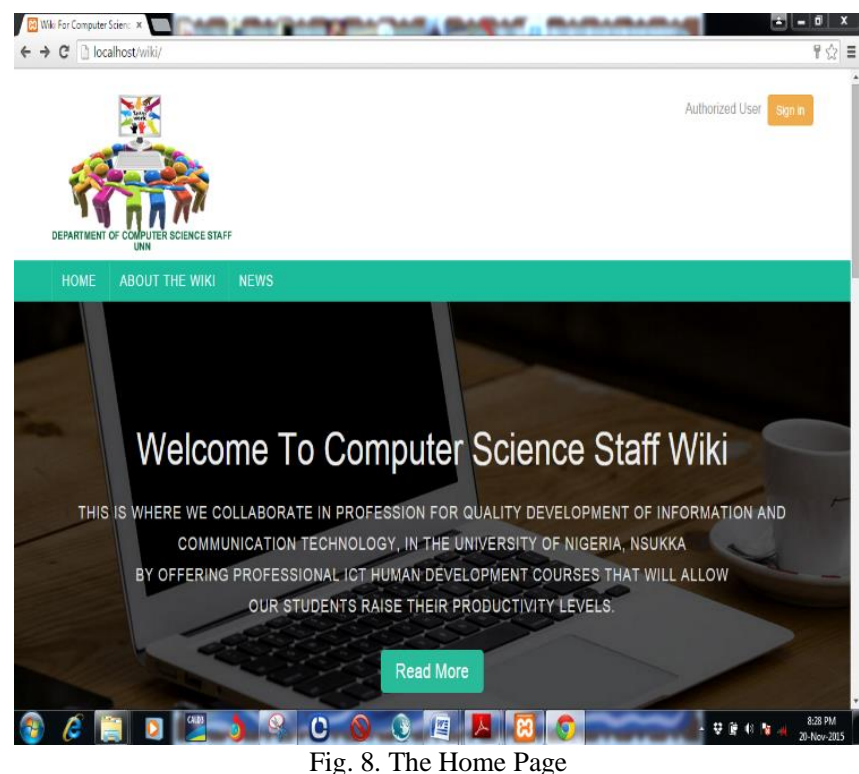

On the login page as shown in Fig. 9, it is expected that the user accessing the page has been properly registered by the system administrator and has obtain an email address which is to be used as the username and a password key from the admin. And when the correct email address and password key are entered, the user is taken to his or her supposed user page, on which the user can enjoy the facilities the system provides therein. 


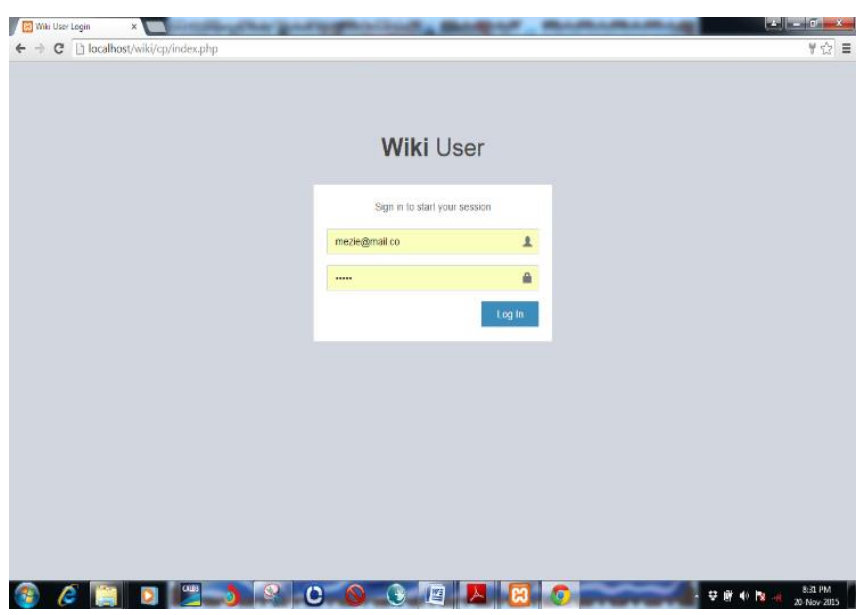

Fig. 9. The User Login Panel.

The Fig. 10 shows the admin page of the system. On this page, the users' registration forms are being prompted when new user is required to be registered in the system. Both the admin and other categories of users are registered here, still by the already existing admin.

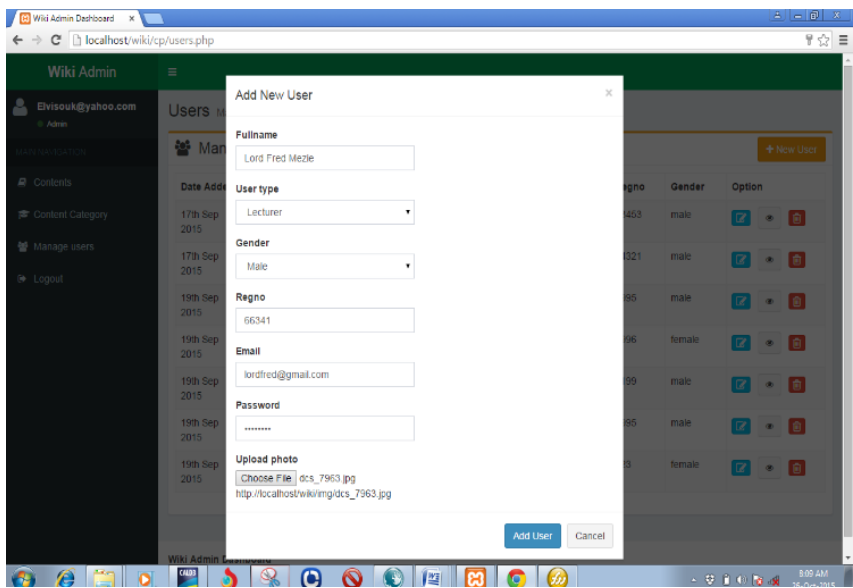

Fig. 10. The User Registration Panel.

Fig. 11 presents the users' management platform of the system. This is only accessible to the admin category of the users. User information update are made here, likewise the deletion of existing users.

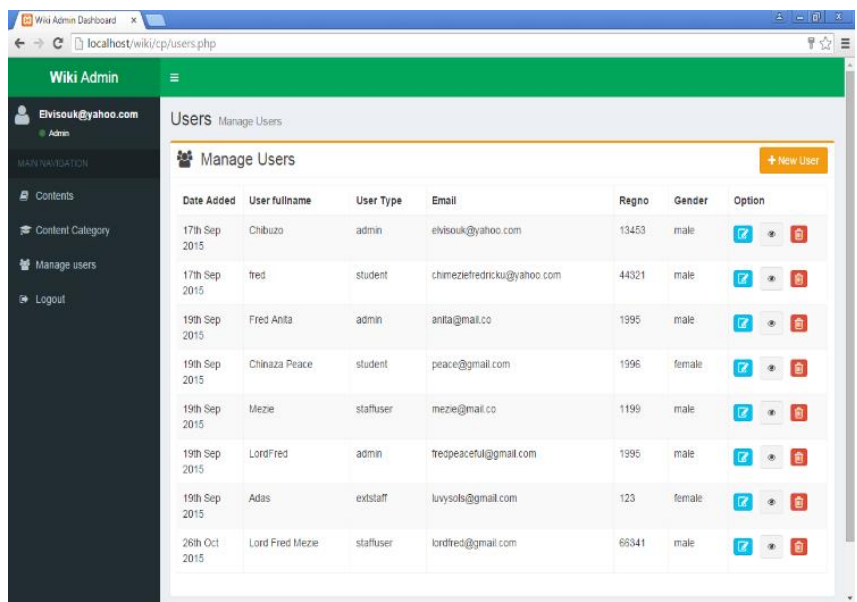

Fig. 11. The Registered Users' Panel for User Management.

Fig. 12 below shows the individual user's profile page. This is where a user can access his or her profile page.
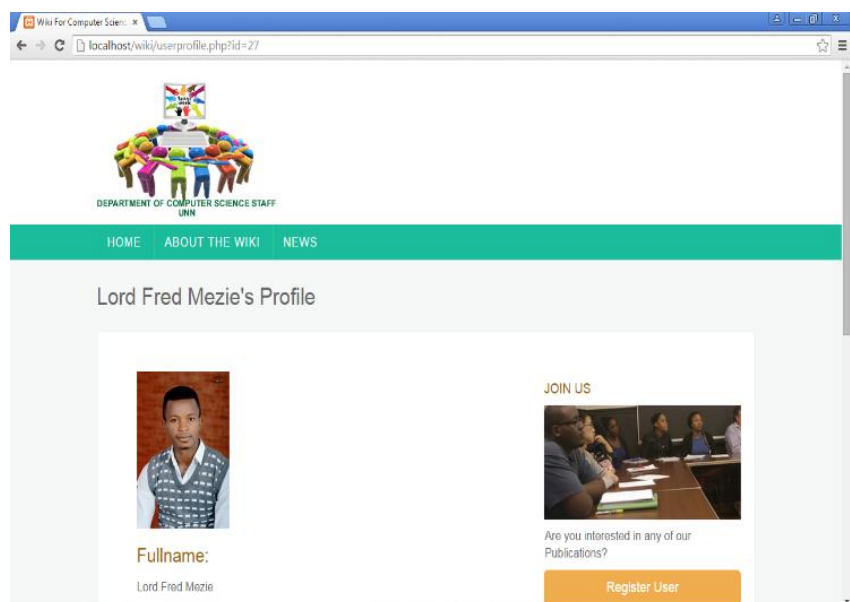

Are you intersted in ary of ou

ord Frod Meze

Fig. 12. The User's Profile Page of the Last User Registered.

Fig. 13 shows the page on which a user could post content in the system. This page prompts a form with which the user makes his or her post whenever the need arises.

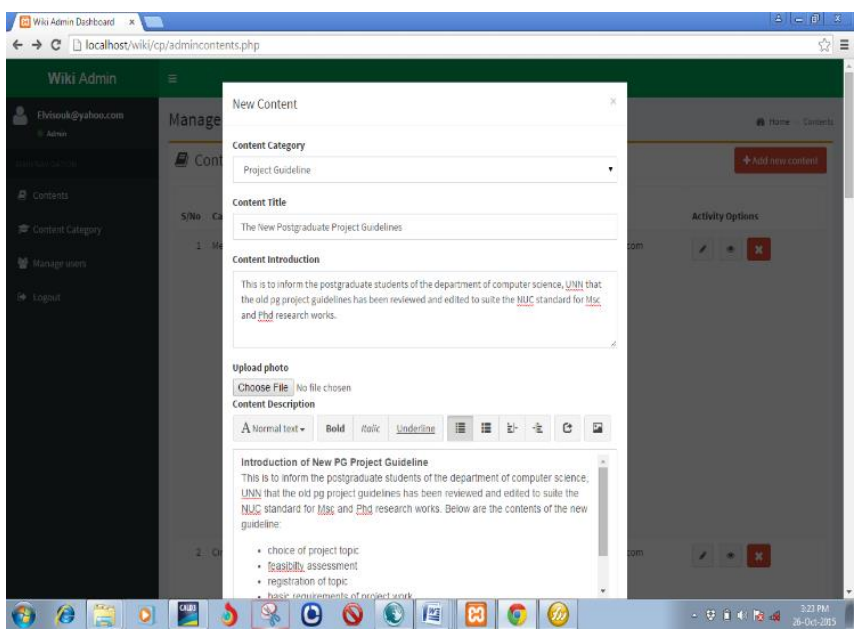

Fig. 13. The Content Post.

This Fig. 14 below shows in brief the content posted by a user with few information about the user. The management operations of the contents posted could be carried out here on this page.

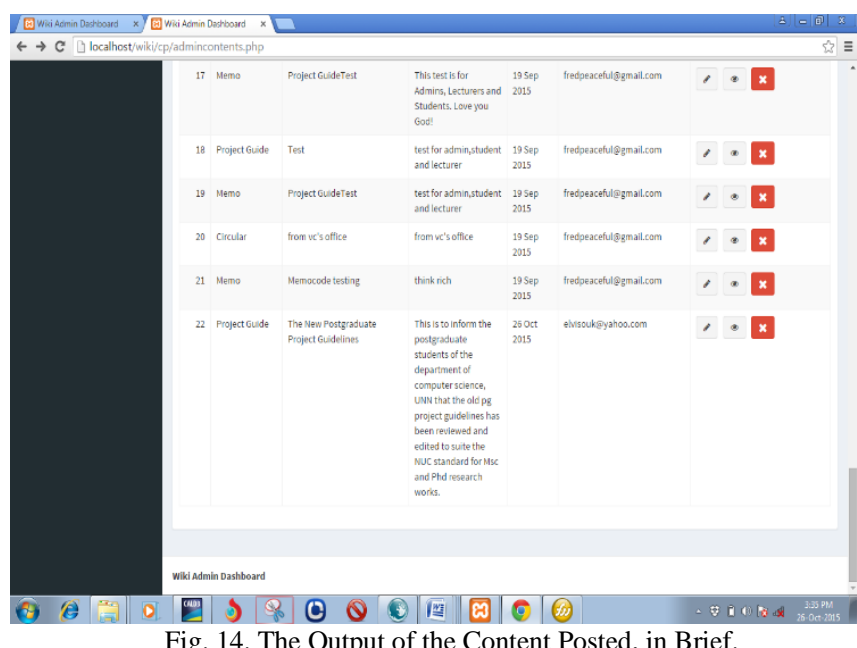

In the Fig. 15 below, the full details of the post could be read, as it present full description of what the content posted 
is all about alongside the picture of the user that made the post.

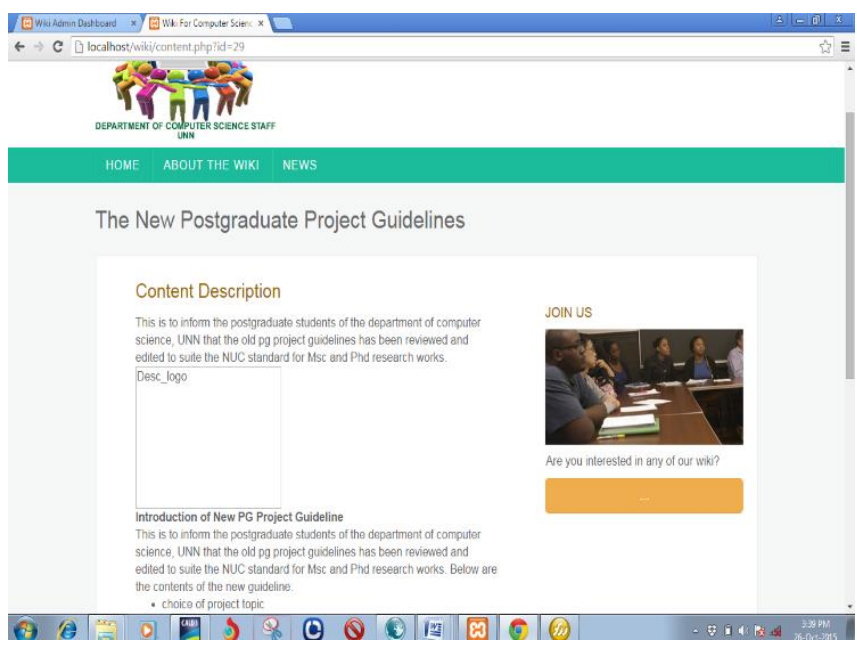

Fig. 15. The Output of the Content Posted, in Detail.

\section{CONCLUSION}

For better throughput and quality of service delivery which are expected from the staff of the Department of Computer Science UNN, the Intranet-Based Wiki with Instant Messaging Protocol have been developed. This is to encourage high level of collaboration and cooperation among the staff of the Department. The system developed in this research helps the staff to respond to their jobs when urgent responses are needed on such work to avoid damages that could be caused by delays. This was achieved by the employment of the Short Message Transfer Protocol (SMTP) via which the system sends instant email messages to the supposed users whenever the admin(s) post(s) a content or document on the system. The developed system has been designated to be hosted in a Virtual Private Server (VPS) on a Virtual Private Network. This is to prevent hackers from attacking the system and it is by this that the system is made Intranet-based.

The achievement made in the development of this research could benefit a lot of organizations. The system is recommended for use in any organization with higher dependency on intelligent staffs with just but a little adjustment or modification.

Other areas that could be needing research attention as regards the "intranet" and "wiki" are; "the employment of wiki in lecturing students on a distant learning programme", "the use of a collaboration conducive system in administering medical services to patients in hospitals by medical doctors", "the importance of a collaboration system for policy development in Government", etc. And all of which must include an instant messaging system to help increase the level of user participation.

\section{REFERENCES}

[1] O. Mansour and L. Askenäs, "Wiki-based Community Collaboration in Organizations".

[2] K. R. Parker, J. Chao, and R. F. Houghton, "Wikis as a Collaboration Tool," 2019.

[3] Deborah Ginsberg, A Wiki, Wiki (Quick) Introduction to the Wide World of Wikis. Available at:
www.aallnet.org/products/pub_sp0607/pub_sp0607_ProDev.pdf\&sa= U\&ved=0CBsQFj, 2006

[4] Bradley Mitchel, "Intranet." Available at: www.compnetworking.about.com/cs/intranets/g/bldef_intranet.htm, 2015.

[5] Danielle Bibbo, James Michelich, Eric Sprehe, Young Eun Lee, "Employing Wiki for knowledge management as a collaborative information repository", an NBC universal case. Journal of Information Technology Teaching Cases. Vol. 2, pp. 17-28, 2012.

[6] Miloš Milovanović, Miroslav Minović, Velimir Štavljanin, Marko Savković \& Dušan Starčević, Wiki as a corporate learning tool, case study for software development company - Behaviour \& Information Technology - Vol. 31, Issue 8, pp. 767-777, 2012.

[7] Jennifer Gonzalez-Reinhart, Wiki and the Wiki Way: Beyond a Knowledge Management Solution, pp. 5-6, 2005.

[8] Benedikt K"ampgen, Basil Ell, Elena Simperl, Denny Vrande “ci'c, Frank Dengler, Enterprise Wikis: Technical Challenges and Opportunities - KIT. Available at:

www.aifb.kit.edu/images/a/ab/Enterprise_wikis_wm2011_kaempgen. pdf\&sa $=$ U\&ved $=0,2011$.

[9] Mansour Osama, Abusalah Mustafa, Askenäs Linda (2011). Wiki collaboration in organizations: An exploratory study. Available at: www.divaportal.

org/smash/get/diva2:413005/fulltext01.pdf\&sa=u\&ved=0ccqqfjajahu kewiwgddd 9 objahuezrqkhqktbsq\&sig2= pmdco $8 \mathrm{mxr} 9 \mathrm{~g} 1 \mathrm{kyfphufgw} \& \mathrm{usg}=$ afqjenewppqstcgxrlprzkmshrlhocyw g, pp. 7, 2011.

[10] Stenmark Dick, "Knowledge Sharing on a Corporate Intranet: Effects of Re- Instating Web Authoring Capability". ECIS 2005 Proceedings. Paper 30. Available at: http://aisel.aisnet.org/ecis2005/30, 2005.

[11] Lester J. Holtzblatt, Laurie E. Damianos, Daniel Weiss, Factors impeding Wiki use in the enterprise. Available at: www.parc.com/content/events/attachments/FactorsImpedingEnterpris eWikiUse_cscw.pdf\&sa=U, 2009.

[12] Stenmark Dick (2007). Knowledge Sharing Through Increased User Participation on a Corporate Intranet. Available at: www.citeseerx.ist.psu.edu/viewdoc/download?doi=10.1.1.380.6802\& rep=rep1\&type=pdf, 2007.

[13] Gbola Olasina, ICT Support for Knowledge Management in Primary Healthcare: A Case Study of Partnership for Reviving Routine Immunization in Northern Nigeria (PRRINN). Available at www.webpages.uidaho.edu/ mbolin/olasina.pdf $\&$ sa $=\mathrm{U} \& v e d=0 \mathrm{CCgQ}$ FjAIahUKEwi_v5mw9YbJAh.

VEtxQKHaP5DoI\&sig2=mczZs2enYzErKJj_odHhZA\&usg=AFQjC NEv10OIRV87IN5MTXktsl4hfSY4gA, 2012.

[14] Michel Buffa, "Intranet Wikis", Proceedings of intra-web workshop. Available at: wwwsop.inria.fr/acacia/WORKSHOPS/IntraWebs2006/Buffa_Intrawebs20 06.pdf. 2006.

[15] Ukekwe Emmanue C. "Development of Wiki for an Academic Department," M.Sc. Project, Computer Science Department, University of Nigeria, Nsukka, 2013.

[16] Peter Dungan, Cathal Heavey (2010). Proposed Visual Wiki System for Gathering Knowledge About Discrete Event Systems. Available at: www.informssim.

org/wsc10papers/046.pdf\&sa=U\&ved=0CBoQFjAGahUKEwipssXe 4bJAhUFMho

KHT5ADoY\&sig2=0ynZBvWZsQWf8muvr41I0A\&usg=AFQjCNFa z6UMmoZdvNpANWNIN8hJ3khhg, 2010.

[17] Katja Hilska-Keinänen, Wiki as an Intranet. Available at: www.eahil.eu/journal/journal_2009_vol5_n2.pdf\#page=8, 2009.

[18] Toby Ward, "Intranet planning: An intranet model for success," Available at: www.prescientdigital.com/articles/intranet-planning-anintranet-model-for-success, 2015

[19] Satish Mishra. Visual modeling \& unified modeling language (uml) Available at: www2.informatik.hu-berlin.de/ hs/Lhre/2004 WS_SWQS/20050107_Ex_UML.ppt.

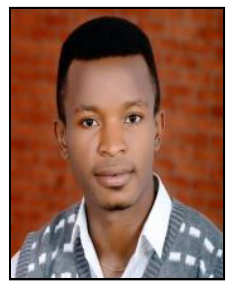

Chimezie F. Ugwu holds a Bachelor of Science degree in Computer Science and Statistics from the University of Nigeria Nsukka. Master of Science Degree in Computer Science at the University of Nigeria, Nsukka and he is currently pursuing his $\mathrm{Ph} . \mathrm{D}$. at Ebonyi State University, Nigeria. He is a Lecturer in the Department of Computer Science, Institute of Management and Technology, IMT Enugu, Nigeria. His area of research interest includes: Ethical hacking and Network Security. 


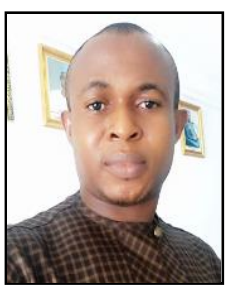

Henry O. Osuagwu obtained his B.Eng. Electronic Engineering and M.Eng. Communication Engineering from University of Nigeria, Nsukka, Nigeria in 2011 and 2016 respectively. He is currently a $\mathrm{Ph} . \mathrm{D}$. student in the Department of Electronic Engineering, University of Nigeria, Nsukka, Nigeria. He is a member of Nigerian Society of Engineers (NSE) and a registered COREN engineer. $\mathrm{He}$ is a Lecturer in the Department of Electrical Engineering, Nigeria Maritime University, Okerenkoko, Delta State, Nigeria. His research interests include Wireless Communication, Radio Resource Management, Network Security, and Cognitive Radio in Wireless Sensor Networks.

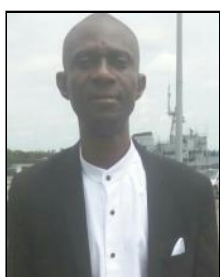

Donatus I. Bayem received his B.Eng. degree in Electrical/Electronics Engineering from Ambrose Alli University, Ekpoma, Nigeria in 1998, and MIT degree from University of Lagos, Nigeria, in 2016. In 2000, he worked at NEET Panasonic Ltd, Port Harcourt as Head, Service Center Engineer and in 2002 worked at COSCHARIS TECHNOLOGIES Ltd Port Harcourt as Sales Engineer. In 2003, he worked with ACTI-TECH Ltd, Abuja-Nigeria as Resident Engineer. In May 2005, he worked at Halogen Security Company Ltd, as Senior Information Technologies Analyst to set up Technologies department and in 2018, he worked at Real Strikers Security Services Ltd as Head, ICT \& Protocol Services. He was involved in severally developing and executing turnkey projects for clients comprising, multinational companies, Government parastatals, Banks, Schools, Telcos, and oil industries. Currently, he is a Lecturer in the Department Electrical Engineering, Nigeria Maritime University, Okerenkoko, Delta State, Nigeria.

$\mathrm{He}$ has certificate in Project Management from Beni American University and Diploma certificate from Axis communications Academy, South Africa. He has Cisco Certified Network Associate (CCNA) in 2011 and Microsoft Certified Technical Specialist (MCTS) in 2011. 